\title{
Enasidenib drives human erythroid differentiation independently of isocitrate dehydrogenase 2
}

\author{
Ritika Dutta, ${ }^{1,2}$ Tian Yi Zhang, ${ }^{1,2}$ Thomas Köhnke, ${ }^{1}$ Daniel Thomas, ${ }^{1}$ Miles Linde, ${ }^{1}$ Eric Gars, ${ }^{1}$ Melissa Stafford, ${ }^{1}$ Satinder Kaur, \\ Yusuke Nakauchi, ${ }^{1}$ Raymond Yin, ${ }^{1}$ Armon Azizi, ${ }^{1}$ Anupama Narla, ${ }^{3}$ and Ravindra Majeti ${ }^{1,2}$ \\ 'Department of Medicine, Division of Hematology, Cancer Institute, and Institute for Stem Cell Biology and Regenerative Medicine, Stanford University, Stanford, California, USA. ${ }^{2}$ Stanford School of \\ Medicine, Stanford, California, USA. ${ }^{3}$ Department of Pediatrics, Division of Hematology/Oncology, Stanford University, Stanford, California, USA.
}

\begin{abstract}
Cancer-related anemia is present in more than $60 \%$ of newly diagnosed cancer patients and is associated with substantial morbidity and high medical costs. Drugs that enhance erythropoiesis are urgently required to decrease transfusion rates and improve quality of life. Clinical studies have observed an unexpected improvement in hemoglobin and RBC transfusionindependence in patients with acute myeloid leukemia (AML) treated with the isocitrate dehydrogenase 2 (IDH2) mutantspecific inhibitor enasidenib, leading to improved quality of life without a reduction in AML disease burden. Here, we demonstrate that enasidenib enhanced human erythroid differentiation of hematopoietic progenitors. The phenomenon was not observed with other IDH1/2 inhibitors and occurred in IDH2-deficient CRISPR-engineered progenitors independently of D-2-hydroxyglutarate. The effect of enasidenib on hematopoietic progenitors was mediated by protoporphyrin accumulation, driving heme production and erythroid differentiation in committed CD71+ progenitors rather than hematopoietic stem cells. Our results position enasidenib as a promising therapeutic agent for improvement of anemia and provide the basis for a clinical trial using enasidenib to decrease transfusion dependence in a wide array of clinical contexts.
\end{abstract}

\section{Introduction}

Acute myeloid leukemia (AML) remains one of the most difficult cancers to treat, with a low cure rate $(25 \%)$ and a 5-year survival rate of $28 \%(1,2)$. High-throughput sequencing of AML patient samples has identified mutations, including FMS-like tyrosine kinase 3 (FLT3) and isocitrate dehydrogenase 1/2 (IDH1/IDH2), for which targeted therapies have been developed. Enasidenib is an FDA-approved, first-in-class agent that preferentially inhibits IDH2-mutant activity and reduces levels of the oncometabolite D-2-hydroxyglutarate (D-2-HG), allowing for differentiation of IDH2-mutated blasts (3-5). Patients with IDH2-mutant AML demonstrated promising clinical responses with enasidenib, leading to its FDA approval in 2017 for IDH2-mutant AML. Notably, patients who did not develop objective responses also derived clinical benefit from enasidenib. Most strikingly, more than $50 \%$ of enasidenib-treated patients who did not demonstrate a decrease in their blast count exhibited improvements in their peripheral blood counts and reached RBC transfusion independence (6). The mechanism underlying this phenomenon is unknown, but is of great clinical interest given the high transfusion-dependence and anemia-associated complications universally associated with AML and other cancers (7). Thus, we sought to investigate how enasidenib drives normal hematopoiesis independent of its effects on AML blast differentiation to improve quality of life and reduce morbidity in cancer-related anemia.

Conflict of interest: RM is a founder, equity holder, and board member of Forty Seven Inc. Copyright: @ 2020, American Society for Clinical Investigation.

Submitted: September 16, 2019; Accepted: December 23, 2019; Published: March 3, 2020. Reference information: J Clin Invest. 2020;130(4):1843-1849.

https://doi.org/10.1172/JCI133344.

\section{Results and Discussion}

We first investigated the effects of enasidenib on normal erythroid differentiation of $\mathrm{CD} 34^{+}$hematopoietic stem and progenitor cells (HSPCs) isolated from umbilical cord blood (CB-CD34+), and identified enhanced differentiation, indicated by an increase in the percentage of $\mathrm{CD}^{+} 1^{+} \mathrm{GPA}^{+}$cells after culture with $\mathrm{EPO}, \mathrm{SCF}$, and IL-3 (erythroid differentiation culture [EDC]) for 8 days (Figure 1A left panel; and Supplemental Figure 1, A and B; supplemental material available online with this article; https://doi.org/10.1172/ JCI133344DS1). CB-CD34+ cells from different donors had a wide range of baseline differentiation capacity. Enasidenib was especially effective at increasing differentiation in $\mathrm{CB}-\mathrm{CD} 34^{+}$cells that had lower baseline differentiation capacity $\left(<40 \% \mathrm{CD}^{\circ} 1^{+}\right.$ $\mathrm{GPA}^{+}$), with a 2.5-fold increase in the proportion of differentiated cells compared with control (Figure 1A, middle and right panels). Enasidenib increased both the proportion and total number of $\mathrm{CD}^{+} 1^{+} \mathrm{GPA}^{+}$cells (Figure 1, A and B) without any decrease in cell viability (Supplemental Figure 2). This increase in erythroid differentiation was dose-dependent and observed at a range of doses from 1-25 $\mu \mathrm{M}$ (Figure 1C). Similar results were observed in CD34 ${ }^{+}$ cells isolated from normal human bone marrow (Figure 1D and Supplemental Figure 1C), as well as in the IDH2-WT TF-1 erythroleukemic cell line (Supplemental Figure 1D and Supplemental Figure 4B). No increase in myeloid differentiation (percentage of $\mathrm{CD}_{3} 3^{+} \mathrm{CD} 14^{+}$) was observed in treated cells (Supplemental Figure 3), indicating that enasidenib selectively promotes erythroid differentiation. The enasidenib-mediated increase in percentage of $\mathrm{CD}^{+} 1^{+} \mathrm{GPA}^{+}$cells was dependent on EPO signaling and did not occur in the absence of the erythroid differentiationpromoting cytokines (Supplemental Figure 4). In the presence of $\mathrm{EPO}$, enasidenib induced a gene expression signature character- 
A

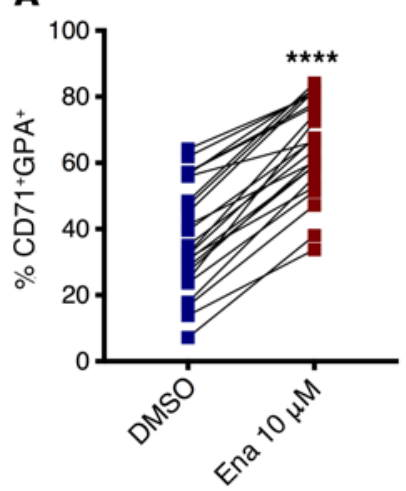

C

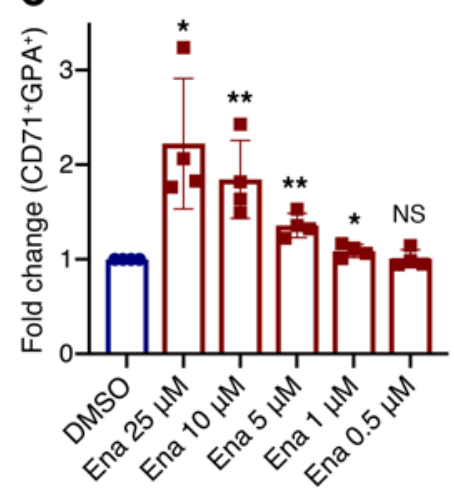

$\mathbf{F}$

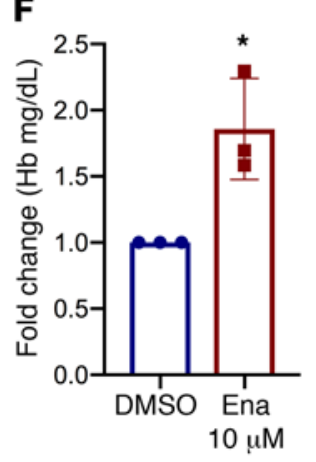

G

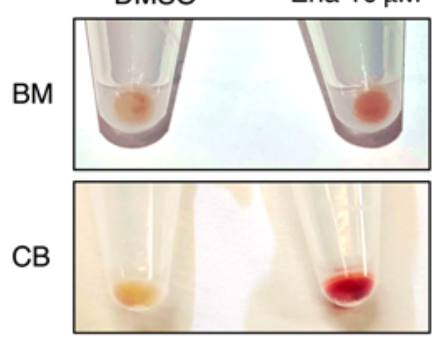

Above $40 \%$

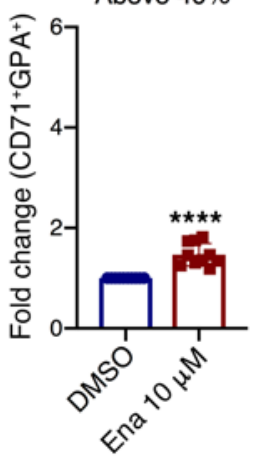

D
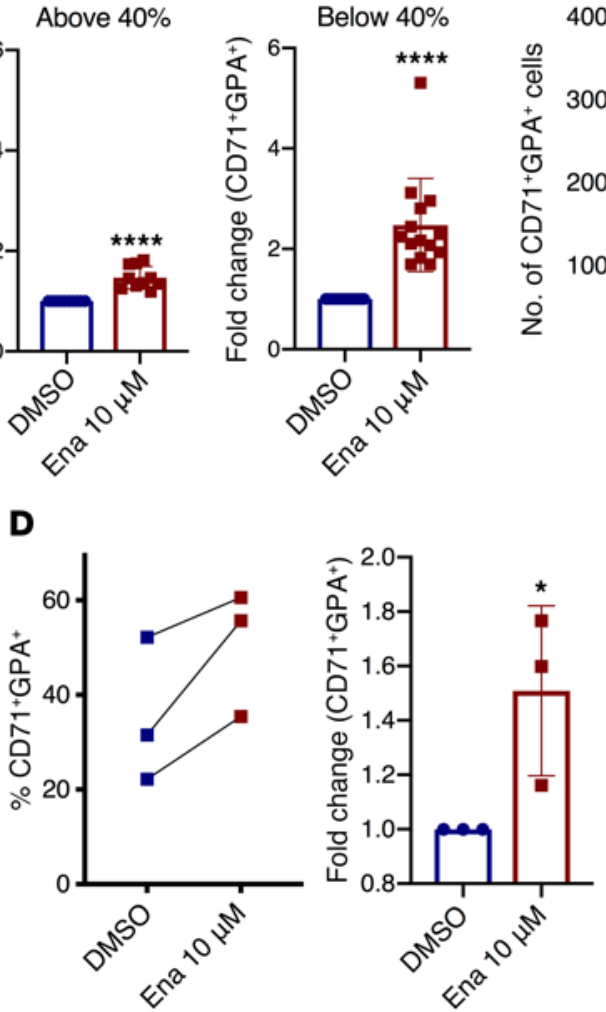

B

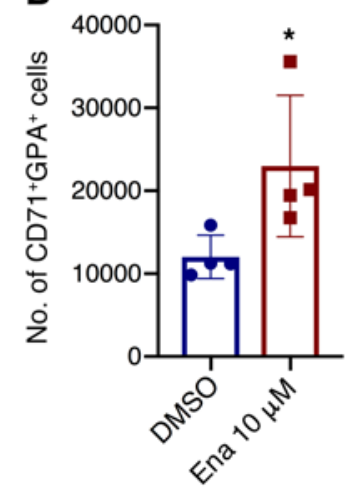

E

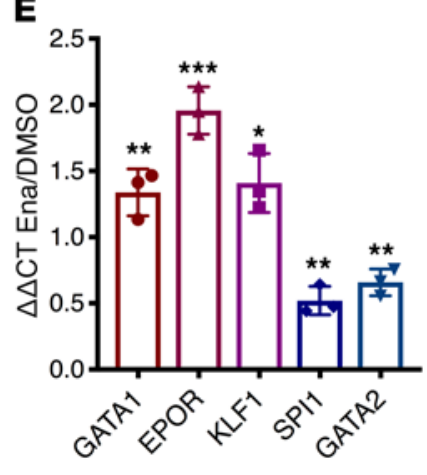

H
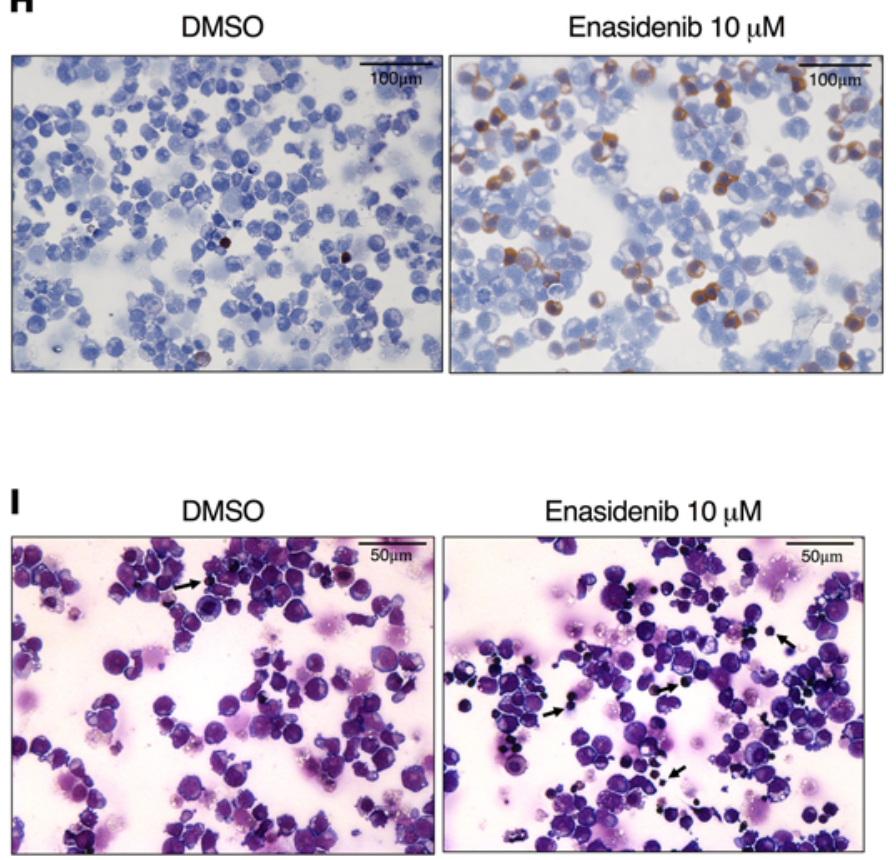

Figure 1. Enasidenib augments erythroid differentiation. (A) Proportion of $C D 71^{+} C P A^{+}\left(\% C D 71^{+} C P A^{+}\right)$cells after 8 days culture of CB-CD34+ cells in EDC with DMSO or $10 \mu \mathrm{M}$ enasidenib (Ena) (left; $n=24$ independent CB specimens). Fold change (FC) of percentage of CD71+CPA ${ }^{+}$cells (DMSO $=1$ ) cells with baseline differentiation capacity (\%CD71+CPA+) of less than $40 \%$ (right; $n=14$ ) or greater than $40 \%$ (middle; $n=10$ ). (B) Number of CB-derived CD71+CPA+ cells at day 8 of $\operatorname{EDC}(n=4)$. (C) Dose response of enasidenib, represented as FC of percentage of CD71+CPA+ cells $(D M S O=1)$ at day 8 of EDC $(n=4)$. (D) Proportion of CD71+CPA ${ }^{+}$cells at day 8 of EDC of CD34+ cells from normal bone marrow (BM) (left; $n=3$ ). FC of percentage of CD71+CPA cells (DMSO $\left.=1\right)($ right; $n=3)$. (E) qPCR detection of relative RNA expression of erythroid and myeloid transcription factors with enasidenib treatment compared with DMSO of CB-CD34+ cells at day 8 of EDC $(D M S O=1)(n=3)$. (F) FC of hemoglobin in a colorimetric assay after 14 days in EDC $(D M S O=1)(n=3)$. (C) Representative cell pellets from normal BM (top panel) and CB (bottom panel) after 14 days in EDC $(n=3)$. (H) Representative image at day 8 of CB-CD34+ cells in EDC treated with DMSO or $10 \mu \mathrm{M}$ enasidenib $(n=3)$ and stained with benzidine. (I) Representative image at day 8 of CB-CD34+ cells in EDC treated with DMSO or $10 \mu \mathrm{M}$ enasidenib $(n=3)$ and stained with Wright-Giemsa. Arrows indicate maturing erythrocytes. Graphs represent mean \pm SD. Statistical significance was calculated using unpaired 2-tailed $t$ tests. ${ }^{*} P<0.05,{ }^{* *} P<0.01,{ }^{* *} P<0.001,{ }^{* * *} P<0.0001$. 
istic of maturing erythrocytes (8), with increased expression of GATA1, EPOR, and KLF1, and decreased SPI1 and GATA2 (Figure $1 \mathrm{E}$ and Supplemental Figure 4). Enasidenib-treated progenitor cells further demonstrated increased hemoglobin production (Figure 1, F-H) and morphologic characteristics of increased erythroid maturation, including decreased cell size and nuclear condensation (Figure 1I). Given the important role of HIF1 $\alpha$ in erythropoiesis and IDH1/2 mutant signaling pathways (9-11), we performed differentiation studies in hypoxic conditions and found that enasidenib also drives erythroid differentiation at lowered oxygen tension (Supplemental Figure 5).

The capacity to increase erythroid differentiation was unique to enasidenib in the class of IDH inhibitors, as AG-120 (a mutant IDH1 inhibitor), AGI-6780 (a mutant IDH2 inhibitor), and AG-881 (a dual mutant IDH1 and IDH2 inhibitor), did not exhibit the same effects at a range of doses from 1-10 $\mu \mathrm{M}$ (Figure 2A). As expected, enasidenib, AGI-6780, and AG-881 completely suppressed D-2HG in a THP-1 cell line overexpressing mutant IDH2-R140Q (Supplemental Figure 6, A and B). To explore whether the effect of enasidenib on erythroid differentiation was mediated through D-2-HG, we measured D-2-HG levels in the differentiating erythroid progenitors. As expected for normal HSPCs, D-2-HG was not present at detectable levels in either the DMSO or enasidenib-treated conditions (Figure 2B). Furthermore, addition of a cell-permeable derivative of D-2-HG (2R-octyl- $\alpha$-hydroxyglutarate) at either 50 or $200 \mu \mathrm{M}$ did not affect the ability of enasidenib to increase the proportion of $\mathrm{CD}^{+} 1^{+} \mathrm{GPA}^{+}$cells (Figure $2 \mathrm{C}$ ).

Because enasidenib has been demonstrated to show moderate inhibitory activity against WT IDH2 at high doses (3), we sought to determine whether the action of enasidenib on erythroid precursors was due to decreased activity of WT IDH2. IDH2 normally functions in the TCA cycle, converting isocitrate into alphaketoglutarate. Treatment of $\mathrm{CD}_{71^{-}}$and $\mathrm{CD} 71^{+}$erythroid precursors with enasidenib did not significantly alter levels of TCA cycle metabolites or affect flux through the TCA cycle, as measured by liquid chromatography-mass spectrometry (LC-MS) and isotope labeling (Supplemental Figure 7). This indicates that WT IDH2 activity was likely not inhibited at the concentration of enasidenib able to drive erythroid differentiation. To further probe the role of IDH2 in this activity of enasidenib, we used CRISPRCas9/AAV6 to knockout IDH2 in $\mathrm{CB}^{-\mathrm{CD} 34^{+}}$cells (Figure 2D and Supplemental Figure 8) (12). Genomic and protein-level disruption of IDH2 were confirmed by PCR detection of the AAV vector in the endogenous IDH2 locus and Western blot analysis, respectively (Figure 2, E and F). IDH2-KO CB-CD34+ cells exhibited decreased baseline erythroid differentiation compared with control AAVS1-edited cells (Figure 2G). However, similar to WT $\mathrm{CB}-\mathrm{CD} 34^{+}$cells, treatment of IDH2-KO CB-CD34 ${ }^{+}$cells with enasidenib still demonstrated a 3.4-fold increase in percentage of $\mathrm{CD} 1^{+} \mathrm{GPA}^{+}$erythroid cells compared with DMSO treatment (Figure $2 \mathrm{H}$ ). These results indicate that enasidenib augments erythroid differentiation independently of both mutant and WT IDH2.

We next sought to identify the progenitor population that enasidenib acts on to drive erythroid maturation. Enasidenib did not increase the number of BFU-E or CFU-E colonies in colonyforming assays (Figure 3A and Supplemental Figure 9), nor did it increase the percentage of BFU-E and CFU-E progenitors in liq- uid culture after 4 days (Figure 3B and Supplemental Figure 10A) (13). In contrast, enasidenib increased late-stage erythroid differentiation after 8 days (14), with increased percentage of GPA (3.7-fold) and increased percentage of Band- $3^{+}$(2.7-fold) (Figure $3 \mathrm{C}$ and Supplemental Figure 10B). As erythroid cells differentiate, they gain CD71 expression (CFU-E stage) and subsequently acquire GPA (erythroblast stage) (15-18). Consistent with the observation that enasidenib augments differentiation by acting on more mature erythroid progenitors, enasidenib treatment did not affect the percentage of $\mathrm{CD}^{+} 1^{+} \mathrm{GPA}^{-}$cells in the first 4 days of culture, but instead increased the percentage of $\mathrm{CD}^{2} 1^{+} \mathrm{GPA}^{+}$cells from day 5 onward (Figure 3D). Furthermore, at least 72 hours of treatment was required for increased differentiation, as washout of the drug before that time point resulted in no increase in erythroid differentiation (Figure 3E). Seventy-two hours of erythroid culture corresponds to the time point when CD71 expression is gained in differentiating erythroid cells in our assay, providing additional evidence that enasidenib does not have an effect before the acquisition of CD71 (Figure 3F). Treating sorted mature CD71 ${ }^{+}$ erythroid progenitors with enasidenib increased erythroid differentiation compared with DMSO controls, whereas enasidenib treatment of $\mathrm{CD}^{-} 1^{-} \mathrm{mid} /$ low early erythroid progenitors showed no effect (Figure 3G and Supplemental Figure 1A). Inhibition of CD71, the transferrin receptor, by a small molecule inhibitor (ferristatin II) caused a dose-dependent decrease in enasidenib-mediated differentiation (Supplemental Figure 11). Together, these observations provide strong evidence that enasidenib acts on $\mathrm{CD} 71^{+}$ erythroid progenitors to increase late-stage differentiation.

To further elucidate the mechanism of enasidenib-induced erythroid differentiation, we considered whether enasidenib might rapidly induce transcriptional changes in $C D 71^{+}$cells. We conducted RNA-sequencing of sorted $\mathrm{CD} 1^{+}$erythroid progenitors treated with enasidenib or DMSO for 24 hours, a time point at which there were no observable erythroid progenitor differences between DMSO- and enasidenib-treated cells (Supplemental Figure 12A). RNA-seq analysis revealed limited transcriptional changes with early enasidenib treatment (Supplemental Figure 12, $\mathrm{B}$ and $\mathrm{C}$ ), suggesting that enasidenib does not directly drive major transcriptional programs.

Given that CD71 is the transferrin receptor and allows for iron uptake into erythropoietic precursors, we hypothesized that enasidenib may affect erythroid differentiation through modulation of the heme biosynthesis pathway, as iron is a critical component of heme synthesis. Enasidenib is known to inhibit ATP binding cassette subfamily G member 2 (ABCG2) (19), a transporter highly expressed in erythroid progenitors that is responsible for efflux of protoporphyrin IX (PPIX), the direct precursor of heme, from the mitochondrion and the cytosol (20-22). Inhibition of ABCG2 by enasidenib might therefore lead to PPIX accumulation, potentially driving increased production of heme and hemoglobin synthesis, leading to increased erythroid differentiation. To explore this hypothesis, we first confirmed expression of ABCG2 on differentiating erythroid progenitors in our assay and observed no difference in ABCG2 expression between DMSO- and enasidenib-treated cells (Supplemental Figure 13). Consistent with previous data, enasidenib exhibited dosedependent inhibition of ABCG2 activity in $\mathrm{CHO}$ cells expressing human ABCG2 (Figure 4A). Measurement of PPIX fluores- 
A
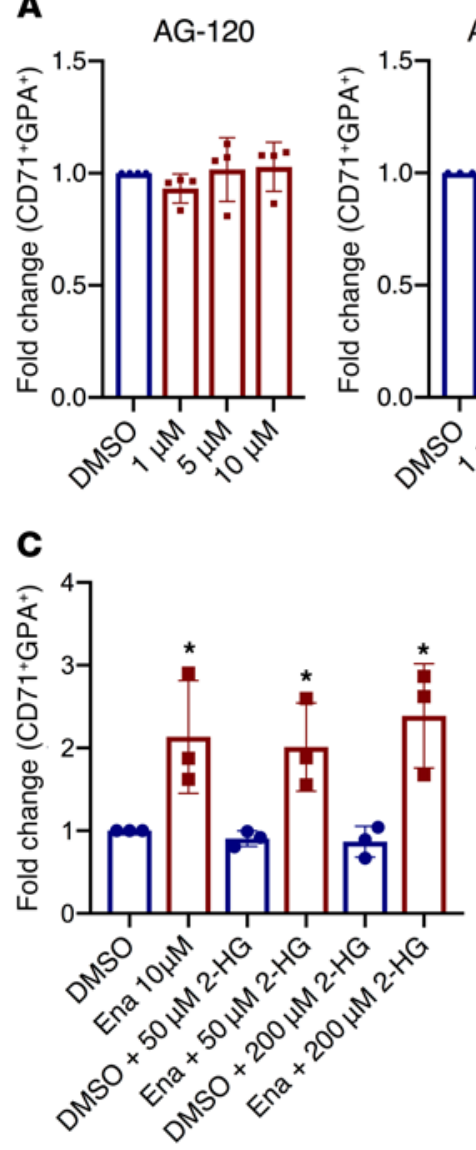

AGI-6780

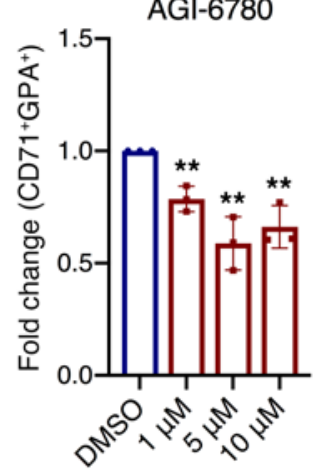

AG-881

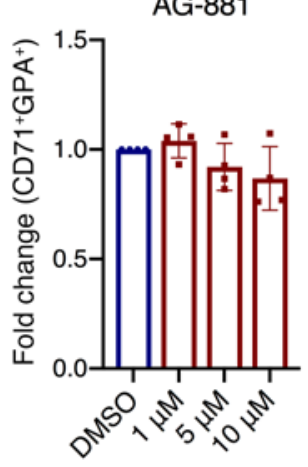

B

D

\section{CRISPR/Cas9 mediated} knock-out of IDH2
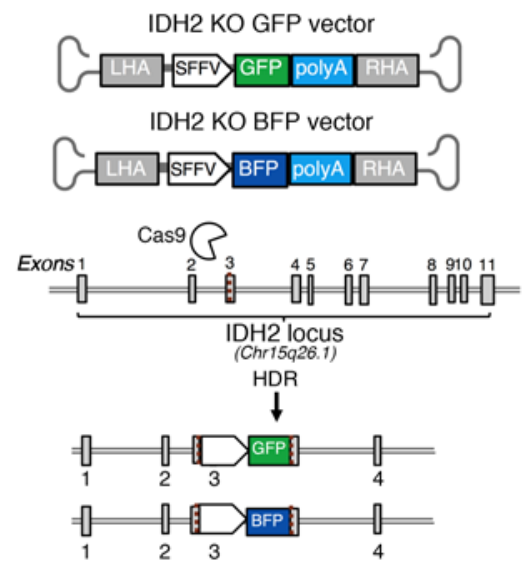

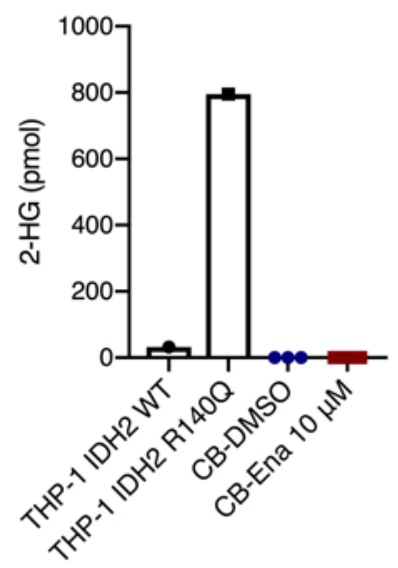

E

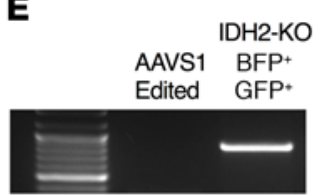

$\mathbf{F}$

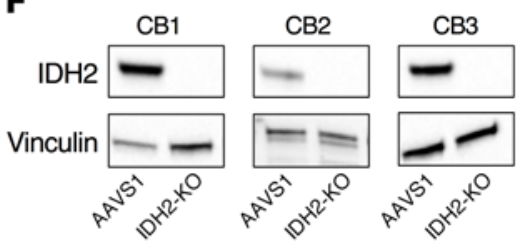

G

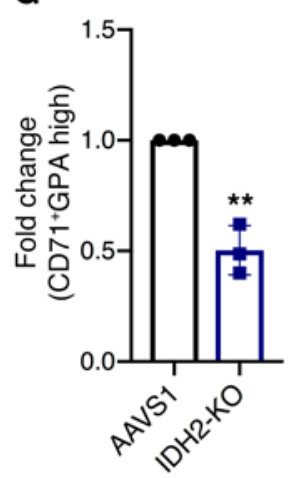

H

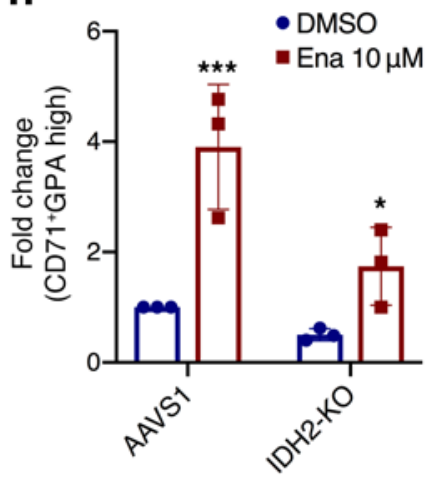

Figure 2. Enasidenib increases erythroid differentiation independently of IDH2. (A) FC of percentage of CD71+GPA+ (DMSO $=1)$ in $\mathrm{CB}-\mathrm{CD} 34^{+}$-derived cells on day 8 of EDC with AG-120 $(n=4)$, AGI-6780 $(n=3)$, and AG-881 $(n=4)$. (B) D-2-HG measurement in the parental THP-1 cell line, an inducible IDH2 R140Q mutant THP-1 cell line, and CB-CD34+-derived cells treated with DMSO or enasidenib for 8 days in EDC $(n=3)$. (C) FC of percentage of CD71+CPA+ (DMSO only $=1)$ in CB-CD34+-derived cells on day 8 of EDC with the addition of (2R)-octyl-alpha-2HG at the indicated concentrations $(n=3)$. (D) Schematic of CRISPR-Cas9 knockout strategy, with disruption of IDH2 in exon 3 and integration of AAV donors with BFP or GFP reporters. RHA/LHA - right/left homology arm (E) PCR with a reverse primer in the AAV donor (SFFV) and forward primer in the genome (IDH2) to confirm site-specific integration of the AAV donor. AAVS1-edited cells (safe harbor locus) were used as control. (F) Western blot showing knockout of IDH2 in 3 independent CB samples, with vinculin as the loading control. (C) FC of percentage of CD71+CPA-high IDH2$\mathrm{KO}$ cells at day 8 of EDC compared with AAVS1 control (AAVS1 $=1)(n=3)$. (H) FC of percentage of CD71+CPA-high in AAVS1 and IDH2-KO cells treated with DMSO or enasidenib (AAVS1 DMSO $=1$, with statistical comparisons made to each respective DMSO condition) $(n=3)$. Cells were gated on live, singlet, $\mathrm{BFP}^{+} \mathrm{GFP}^{+}$before gating on CD71/GPA. Graphs represent mean \pm SD. Statistical significance was calculated using unpaired 2-tailed $t$ tests. ${ }^{*} P<0.05,{ }^{* *} P<0.01,{ }^{* *} P<0.001$. cence by flow cytometry (Figure 4B) and microscopy (Figure 4C) in $\mathrm{CB}-\mathrm{CD} 34^{+}$-derived erythroid progenitors revealed a 1.2 -fold increase of PPIX fluorescence in enasidenib-treated cells compared with DMSO. Ultra-performance liquid chromatography (UPLC) measurement of PPIX showed a 6.1-fold increase in PPIX levels after enasidenib treatment (Figure 4D), along with increased hemin (FePPIX) and ZnPPIX (Figure 4E), a form of PPIX with incorporated zinc instead of iron. ZnPPIX formation is indicative of excess PPIX and is seen in porphyrias with PPIX accumulation (23). This increased PPIX did not increase reactive oxygen species (Supplemental Figure 14A) or oxidative stress, as measured by the GSH/GSSG ratio (Supplemental Figure 14B). To determine if increased PPIX enhances erythropoiesis, we treated CB-CD34 ${ }^{+}$ cells in EDC with $20 \mu \mathrm{M}$ Ko143, a potent ABCG2 inhibitor, and observed a similar increase in the percentage of $\mathrm{CD} 1^{+} \mathrm{GPA}^{+}$cells and PPIX fluorescence, as seen with enasidenib (Figure 4, F and G). 
A

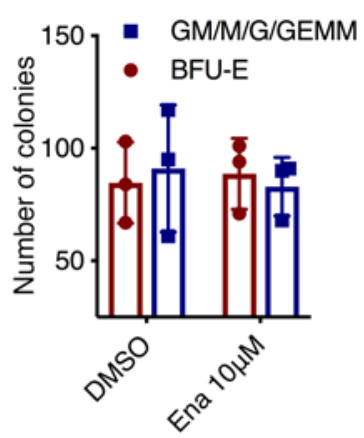

B

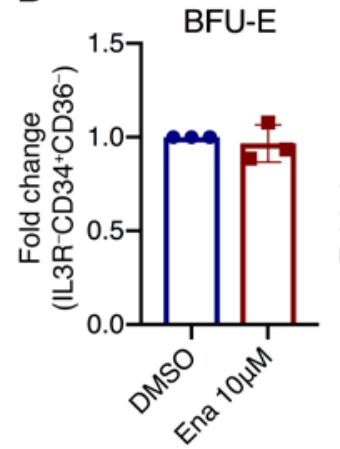

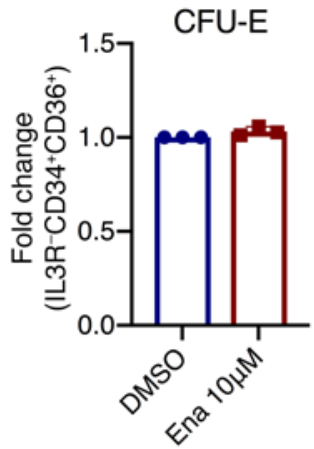

C
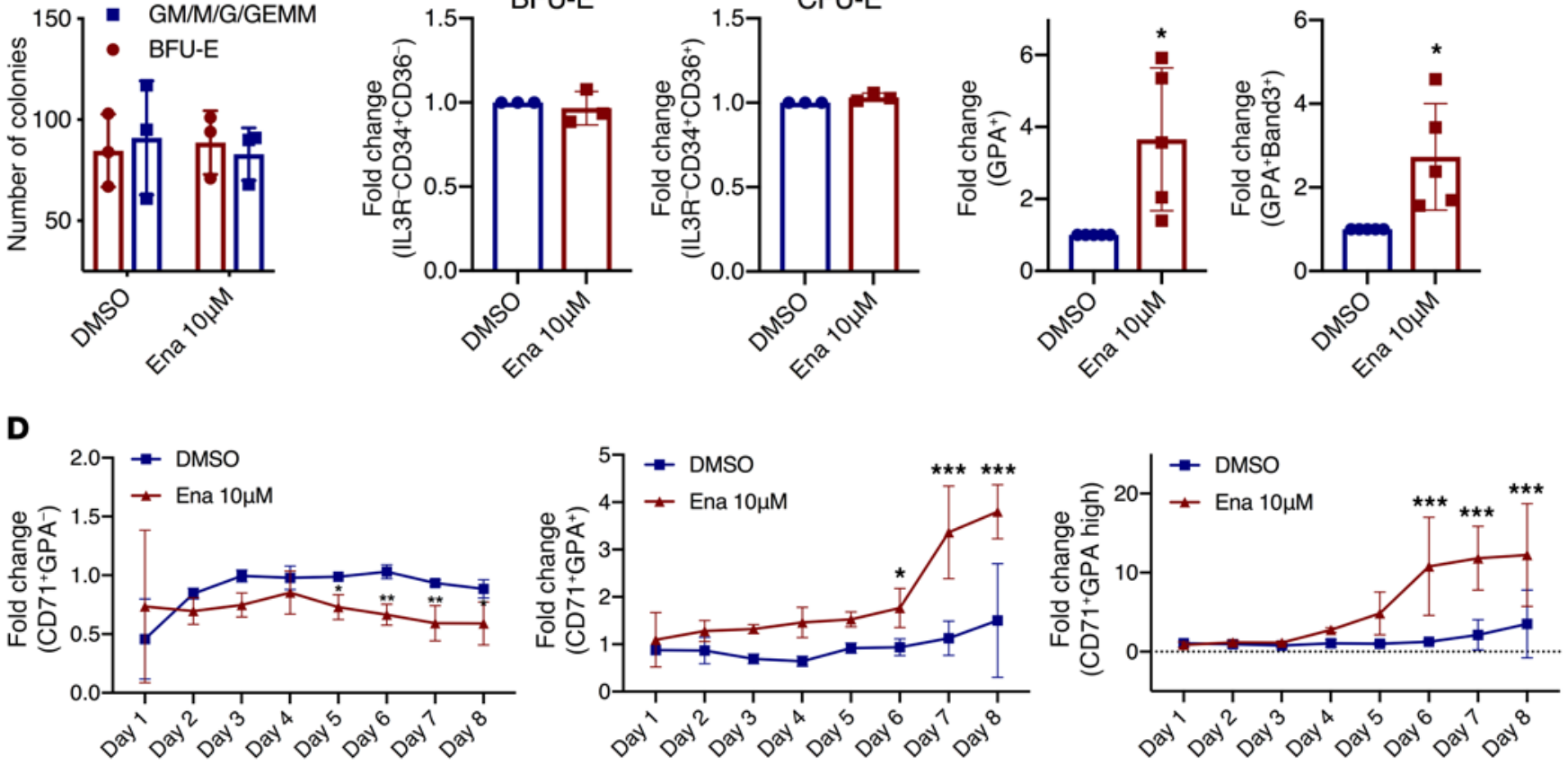

$\mathbf{E}$

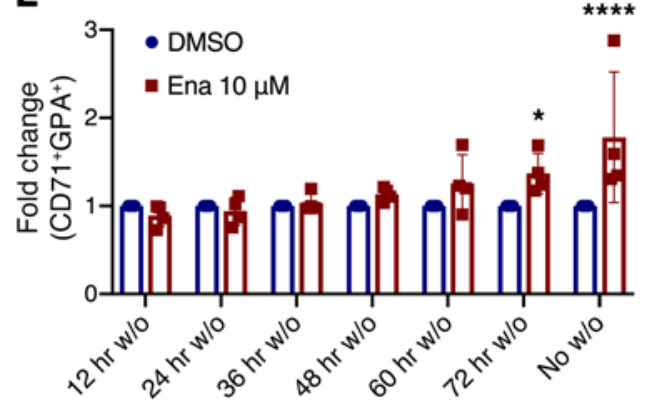

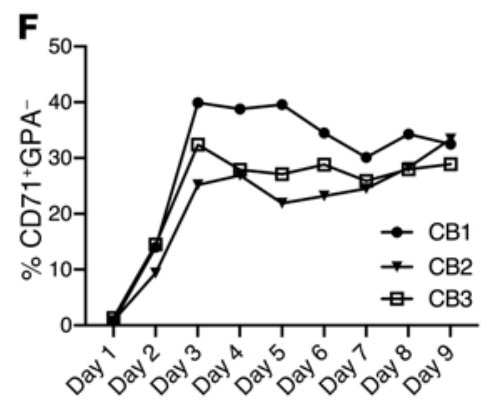

G

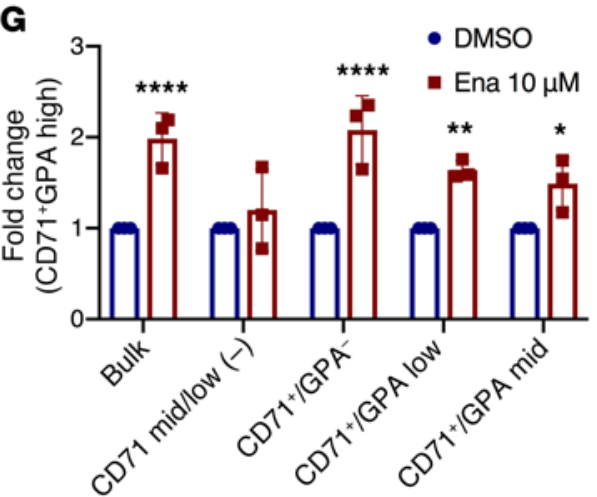

Figure 3. Enasidenib acts on mature $\mathrm{CD71}^{+}$erythroid progenitors to increase differentiation. (A) Methylcellulose colony forming assay of $\mathrm{CB}-\mathrm{CD} 34^{+}$cells indicating the number of erythroid colonies (BFU-E) and myeloid colonies (GM/M/GEMM) observed with DMSO or enasidenib treatment after 14 days $(n=3)$. (B) FC of percentage of BFU-E (IL3R-CD34+CD36-) (middle) and percentage of CFU-E (IL3R-CD34-CD36+) (right) at day 4 of EDC (DMSO = 1) ( $n=3$ ). (C) FC of percentage of GPA $\left(\right.$ left) and percentage of CPA+Band3 $3^{+}$(right) at day 8 of EDC $(D M S O=1)(n=3)$. (D) Time course of erythroid differentiation: FC of percentage of CD71+CPA- (left), percentage of CD71+CPA (middle), and percentage of CD71+CPA-high (right) relative to untreated cells (not shown) ( $n=3$ ). (E) FC of percentage of $C D 71^{+} C P A^{+}$measured at day 8 of EDC, with DMSO or enasidenib washed out $(w / 0)$ of the culture at the indicated time points $(D M S O=1)(n=4)$. (F) Timeline of the gain of CD71 expression (\%CD71+CPA-) in 3 untreated CB samples. (C) Cells were sorted into CD71 mid/low, CD71+CPA-, CD71+CPA-low, and CD71+CPA-mid after 6 days of EDC, and then treated with DMSO or enasidenib for 4 days. FC of percentage of CD71+CPA-high (DMSO for each population $=1$ ) $(n=3)$. Graphs represent mean $\pm \mathrm{SD}$. Statistical significance was calculated using unpaired 2-tailed $t$ tests. ${ }^{*} P<0.05,{ }^{* *} P<0.01,{ }^{* * *} P<0.001,{ }^{* * *} P<0.0001$.

Intracellular heme (ferrous PPIX) drives transcriptional and translational programs that promote coordinated erythroid differentiation through multiple mechanisms, including modulation of Bach1 transcriptional activity and heme-regulated eIF2 $\alpha$-kinase (HRI) kinase activity (24-26). Because heme is a strong driver of globin chain synthesis (25-27), we measured transcription of globin chain genes following treatment with enasidenib by quantitative PCR (qPCR)and observed a striking upregulation of all globin chains, including $H B A 1 / 2$ (23-fold), $H B B$ (8-fold), $H B D$ (4-fold), and HBG1/2 (3.5-fold) (Figure 4H). These results support a model in which ABCG2 inhibition by enasidenib drives PPIX accumulation, leading to increased heme and hemoglobin produc- tion in erythroid progenitors, thereby driving increased erythroid differentiation (Figure 4I).

Symptomatic anemia remains a major clinical problem in cancer patients regardless of mutational context, and contributes to decreased quality of life and increased morbidity. Anemia is also a significant side effect of cancer treatments, ranging from regimens of platinum-based chemotherapy and radiation to novel agents such as azacitadine and venetoclax $(28,29)$. Given that transfusion independence in cancer patients is associated with increased survival, drugs that improve normal hematopoiesis have great potential to reduce morbidity and mortality (30). In this study, we show that enasidenib, an FDA-approved therapy for treatment of 

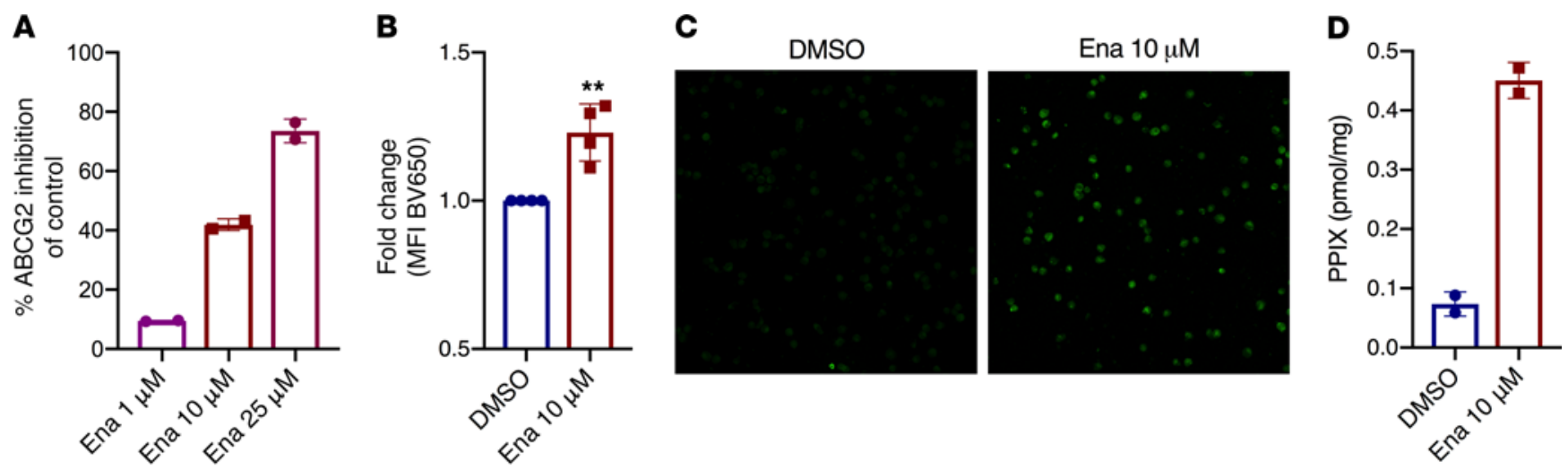

E

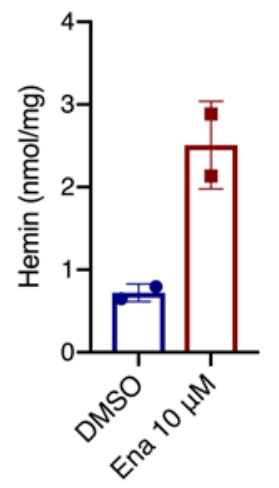

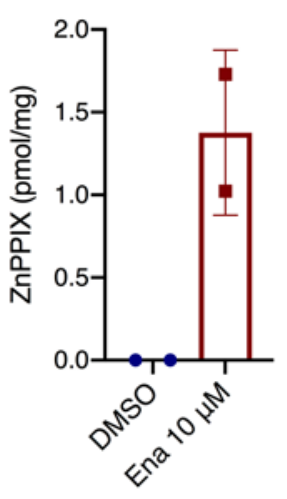

$\mathbf{F}$

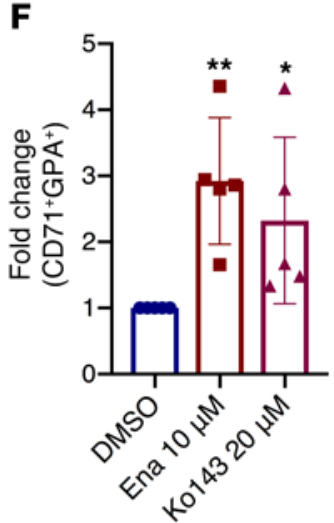

I

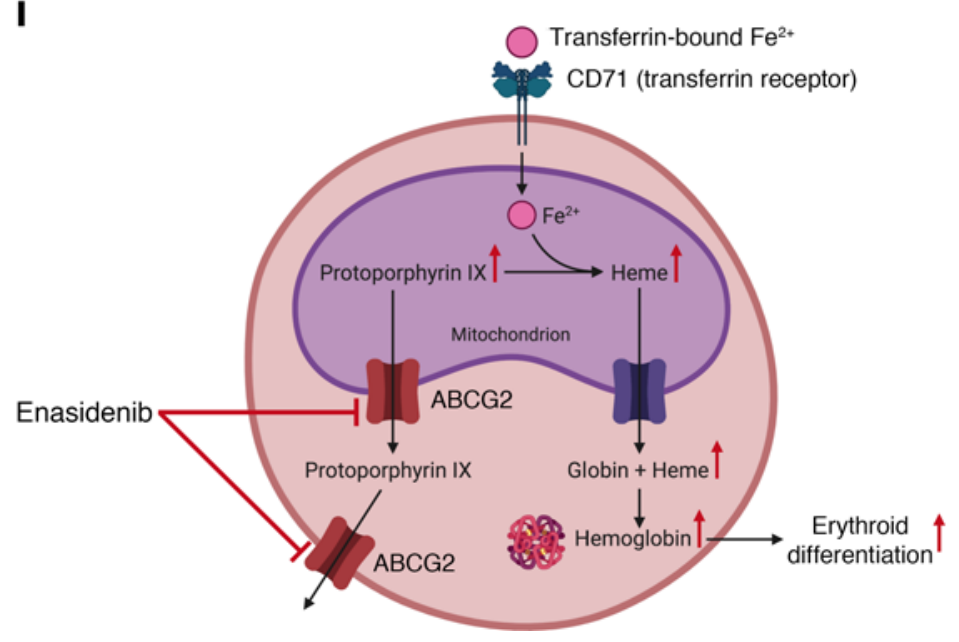

G

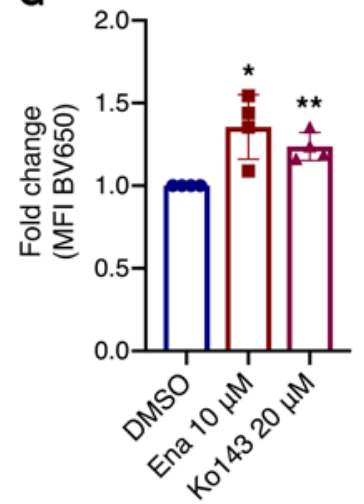

H

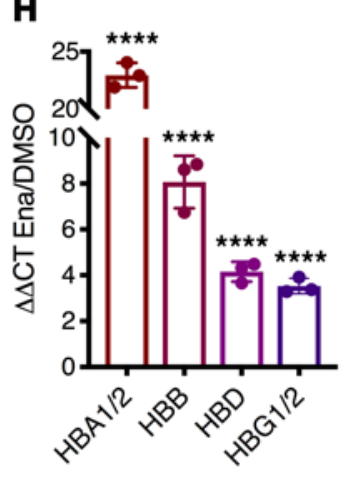

Figure 4. Enasidenib modulates heme biosynthesis through accumulation of PPIX. (A) Enasidenib-mediated inhibition of ABCC2-mediated Hoechst efflux in CHO cells, measured in duplicate, as percentage of inhibition relative to DMSO control. (B) FC of MFI measuring PPIX fluorescence by flow cytometry (BV650 channel) in CB-CD34+-derived cells at D8 of EDC (DMSO $=1)(n=4)$. (C) Representative microscopy image of PPIX (HeNe 633 laser, original magnification $\times 20)$ in CB-CD34+-derived cells at day 8 of $\operatorname{EDC}(n=3)$ (D) UPLC levels of PPIX after 8 days of enasidenib treatment. For $\mathbf{D}$ and $\mathbf{E}$, each point represents 3 independent $\mathrm{CB}-\mathrm{CD} 34^{+}$samples that were pooled together before metabolite measurement. (E) UPLC levels of hemin and $\mathrm{ZnPPIX}$ after 8 days of enasidenib treatment. (F) FC of percentage of $C D 71^{+} \mathrm{CPA}{ }^{+}$cells at day 8 of EDC after treatment with enasidenib or Ko143 (DMSO = 1) $(n=5)$. (C) FC of MFI measuring PPIX fluorescence by flow cytometry (BV650 channel) in CB-CD34+-derived cells at day 8 of EDC with enasidenib or Ko143 $(\mathrm{DMSO}=1)(n=4)$. (H) qPCR determination of relative RNA expression of hemoglobin genes in enasidenib-treated CB-CD34+-derived cells at day 8 of EDC (DMSO $=1)(n=3)$. (I) Schematic of proposed model. Graphs represent mean $\pm \mathrm{SD}$. Statistical significance was calculated using unpaired 2 -tailed $t$ tests. ${ }^{*} P<0.05$, ${ }^{* *} P<0.01$, ${ }^{* * * *} P<0.0001$. relapsed/refractory IDH2-mutant AML, drives erythroid differentiation in $\mathrm{CD}^{+} 1^{+}$erythroid precursors independently of IDH2 through modulation of PPIX homeostasis and hemoglobin production. These novel findings provide a mechanism explaining the previous clinical observations that enasidenib promotes increased hemoglobin levels and RBC transfusion independence in patients with AML, even when blast count is unchanged $(6,31)$. This study is the first to present evidence that enasidenib can potentially be repurposed to treat anemia in any clinical context with functional erythroid precursors. Thus, our results provide the groundwork for a clinical trial using enasidenib for improving anemia and decreasing transfusion burden in a wide range of diseases.

\section{Methods}

See the Supplemental Material for the detailed experimental methods.

Study approval and data availability. Cord blood and normal bone marrow samples were obtained according to protocols approved by the Administrative Panel on Human Subjects Research Institutional Review Board/Stem Cell Research Oversight Panel (IRB/SCRO) (Stanford IRB nos. 36560, 6453, 14839, and SCRO-291) 
with informed consent. RNA-sequencing data are available through GEO (accession number GSE140108).

\section{Author contributions}

RD, TYZ, TK, DT, ML, EG, AN, and RM designed experiments. RD, TYZ, TK, DT, ML, EG, MS, SK, YN, and RY optimized and conducted experiments. RD, TK, AA, and RM analyzed data. RD and RM wrote the manuscript.

\section{Acknowledgments}

We acknowledge the Flow Cytometry Core of the Stanford Stem Cell Institute, the Binns Program for Cord Blood Research, the Stanford Neuroscience Microscopy Service (NIH NS069375), the Iron and Heme Core facility at University of Utah (NIH NIDDK U54DK110858), and Amy Fan for her input. This work was supported by NIH grants (1R01CA188055, R01HL4436, and 1R01HL142637), the Stanford Ludwig Center for Cancer Stem Cell Research and Medicine, and the Leukemia and Lymphoma Society Scholar Award to RM. RD is supported by the Howard Hughes Medical Institute Medical Fellowship award and the Stanford Medical Scholars Program.

Address correspondence to: Ravindra Majeti, Lokey Stem Cell Research Building, Room G3005, 265 Campus Drive, Stanford, California 94305, USA. Phone: 650.721.6376; Email: rmajeti@ stanford.edu.
1. [No authors listed]. SEER Cancer Stat Facts: Acute Myeloid Leukemia. National Cancer Institute. https://seer.cancer.gov/statfacts/html/amyl.html. Accessed February 24, 2020.

2. Shah A, Andersson TM, Rachet B, Björkholm $\mathrm{M}$, Lambert PC. Survival and cure of acute myeloid leukaemia in England, 1971-2006: a population-based study. Br J Haematol. 2013;162(4):509-516.

3. Yen K, et al. AG-221, a first-in-class therapy targeting acute myeloid leukemia harboring oncogenic IDH2 mutations. Cancer Discov. 2017;7(5):478-493.

4. Thomas D, Majeti R. Optimizing next-generation AML therapy: activity of mutant IDH2 inhibitor AG-221 in preclinical models. Cancer Discov. 2017;7(5):459-461.

5. Stein EM, et al. Enasidenib in mutant $I D H 2$ relapsed or refractory acute myeloid leukemia. Blood. 2017;130(6):722-731.

6. Stein EM, et al. Molecular remission and response patterns in patients with mutant- $I D H 2$ acute myeloid leukemia treated with enasidenib. Blood. 2019;133(7):676-687.

7. Cannas G, et al. Transfusion dependency at diagnosis and transfusion intensity during initial chemotherapy are associated with poorer outcomes in adult acute myeloid leukemia. Ann Hematol. 2015;94(11):1797-1806.

8. Ferreira R, Ohneda K, Yamamoto M, Philipsen S. GATA1 function, a paradigm for transcription factors in hematopoiesis. Mol Cell Biol. 2005;25(4):1215-1227.

9. Koivunen $\mathrm{P}$, et al. Transformation by the (R)enantiomer of 2-hydroxyglutarate linked to EGLN activation. Nature. 2012;483(7390):484-488.

10. Losman JA, et al. (R)-2-hydroxyglutarate is sufficient to promote leukemogenesis and its effects are reversible. Science. 2013;339(6127):1621-1625.

11. Haase VH. Regulation of erythropoiesis by hypoxia-inducible factors. Blood Rev. 2013;27(1):41-53.
12. Bak RO, Dever DP, Reinisch A, Cruz Hernandez D, Majeti R, Porteus MH. Multiplexed genetic engineering of human hematopoietic stem and progenitor cells using CRISPR/Cas9 and AAV6. Elife. 2017;6:e27873.

13. Li J, et al. Isolation and transcriptome analyses of human erythroid progenitors: BFU-E and CFU-E. Blood. 2014;124(24):3636-3645.

14 . $\mathrm{Hu}$ J, et al. Isolation and functional characterization of human erythroblasts at distinct stages: implications for understanding of normal and disordered erythropoiesis in vivo. Blood. 2013;121(16):3246-3253.

15. van Lochem EG, van der Velden VH, Wind HK, te Marvelde JG, Westerdaal NA, van Dongen JJ. Immunophenotypic differentiation patterns of normal hematopoiesis in human bone marrow: reference patterns for age-related changes and disease-induced shifts. Cytometry B Clin Cytom. 2004;60(1):1-13.

16. Okumura N, Tsuji K, Nakahata T. Changes in cell surface antigen expressions during proliferation and differentiation of human erythroid progenitors. Blood. 1992;80(3):642-650.

17. Chen K, Liu J, Heck S, Chasis JA, An X, Mohandas $\mathrm{N}$. Resolving the distinct stages in erythroid differentiation based on dynamic changes in membrane protein expression during erythropoiesis. Proc Natl Acad Sci U S A . 2009;106(41):17413-17418.

18. Mao B, et al. Early development of definitive erythroblasts from human pluripotent stem cells defined by expression of glycophorin A/CD235a, CD34, and CD36. Stem Cell Reports. 2016;7(5):869-883.

19. [No authors listed]. IDHIFA (enasidenib) drug information. FDA. https://www.accessdata.fda. gov/drugsatfda_docs/label/2017/209606s0001bl.pdf. Accessed January 28, 2020.

20. Zhou S, Zong Y, Ney PA, Nair G, Stewart CF, Sorrentino BP. Increased expression of the Abcg2 transporter during erythroid maturation plays a role in decreasing cellular protoporphyrin IX levels. Blood. 2005;105(6):2571-2576.

21. Kobuchi H, et al. Mitochondrial localization of ABC transporter ABCG2 and its function in 5-aminolevulinic acid-mediated protoporphyrin IX accumulation. PLoS One. 2012;7(11):e50082.

22. Jonker JW, et al. The breast cancer resistance protein protects against a major chlorophyll-derived dietary phototoxin and protoporphyria. Proc Natl Acad Sci U S A. 2002;99(24):15649-15654.

23. Sachar M, Anderson KE, Ma X. Protoporphyrin IX: the Good, the Bad, and the Ugly. JPharmacol Exp Ther. 2016;356(2):267-275.

24. Chen JJ. Translational control by heme-regulated eIF2 $\alpha$ kinase during erythropoiesis. Curr Opin Hematol. 2014;21(3):172-178.

25. Tahara T, Sun J, Igarashi K, Taketani S. Hemedependent up-regulation of the alpha-globin gene expression by transcriptional repressor Bach1 in erythroid cells. Biochem Biophys Res Commun. 2004;324(1):77-85.

26. Tahara T, et al. Heme positively regulates the expression of beta-globin at the locus control region via the transcriptional factor Bach1 in erythroid cells. J Biol Chem. 2004;279(7):5480-5487.

27. Gross M, Rabinovitz M. Control of globin synthesis by hemin: factors influencing formation of an inhibitor of globin chain initiation in reticulocyte lysates. Biochim Biophys Acta. 1972;287(2):340-352.

28. Kuzur ME, Greco FA. Cisplatin-induced anemia. NEngl JMed.1980;303(2):110-111.

29. DiNardo CD, et al. Venetoclax combined with decitabine or azacitidine in treatment-naive, elderly patients with acute myeloid leukemia. Blood. 2019;133(1):7-17.

30. Gavillet M, Noetzli J, Blum S, Duchosal MA, Spertini O, Lambert JF. Transfusion independence and survival in patients with acute myeloid leukemia treated with 5-azacytidine. Haematologica. 2012;97(12):1929-1931.

31. Pollyea DA, et al. Enasidenib, an inhibitor of mutant IDH2 proteins, induces durable remissions in older patients with newly diagnosed acute myeloid leukemia. Leukemia. 2019;33(11):2575-2584. 